\title{
Impact of Potato + Pigeon Pea based Intercropping Systems on Microbial Properties
}

\author{
M. H. Manjunatha ${ }^{1 *}$, S. Bhaskar ${ }^{2}$, A. Sathish ${ }^{1}$, B. Basavaraja ${ }^{1}$ and N. N. Lingaraju ${ }^{1}$ \\ ${ }^{1}$ Department of Agronomy, UAS, GKVK, Bangalore, India \\ ${ }^{2} A A G C C, I C A R$, New Delhi, India \\ *Corresponding author
}

\section{A B S T R A C T}

\section{Keywords}

Intercropping

Systems, Potato,

Pigeon pea

Article Info

Accepted:

15 January 2021

Available Online:

10 February 2021
A field experiments were conducted on the "Impact of potato + pigeon pea based intercropping systems on microbial properties" in the premises of Agricultural Research Station (ARS), Madenur, Hassan Taluk and District during Kharif and Rabi 2017 and 2018. Experiment consisting of twelve treatments $\left(\mathrm{T}_{1}: 100 \% \mathrm{~K}\right.$ through $\mathrm{K}_{2} \mathrm{SO}_{4}+\mathrm{NP}$ through fertilizers for targeted yield of $20 \mathrm{t} \mathrm{ha}^{-1,} \mathrm{~T}_{2}: 100 \% \mathrm{~K}$ through Bio-K + balance NP through fertilizers for targeted yield of $20 \mathrm{t} \mathrm{ha}^{-1,} \mathrm{~T}_{3}: 50 \% \mathrm{~K}$ through Bio-K $+50 \% \mathrm{~K}$ through $\mathrm{K}_{2} \mathrm{SO}_{4}+$ balance NP through fertilizers for targeted yield of $20 \mathrm{t} \mathrm{ha}^{-1,} \mathrm{~T}_{4}: 100 \% \mathrm{~K}$ through $\mathrm{K}_{2} \mathrm{SO}_{4}+\mathrm{NP}$ through fertilizers for targeted yield of $25 \mathrm{t} \mathrm{ha}^{-1,} \mathrm{~T}_{5}: 100 \% \mathrm{~K}$ through Bio-K + balance NP through fertilizers for targeted yield of $25 \mathrm{t} \mathrm{ha}^{-1,} \mathrm{~T}_{6}: 50 \% \mathrm{~K}$ through Bio-K $+50 \% \mathrm{~K}$ through $\mathrm{K}_{2} \mathrm{SO}_{4}+$ balance NP through fertilizers for targeted yield of $25 \mathrm{tha}^{-1,} \mathrm{~T}_{7}$ : Recommended dose of fertilizers alone (75:75:100 kg NPK ha $\left.{ }^{-1}\right), \mathrm{T}_{8}$ : Package of Practice recommendation, UAS B) and replicated thrice. Among the soil biological properties, microbial activity viz., bacteria, fungi and actinomycetes count after harvest of potato was significantly higher in treatments receiving $100 \% \mathrm{~K}$ through Bio-K + balance NP through fertilizers for targeted yield of $25 \mathrm{tha}^{-1}\left(15.87 \times 10^{6} \mathrm{Cfu} \mathrm{g}^{-1}, 9.53 \times 10^{4} \mathrm{Cfu} \mathrm{g}^{-1}\right.$ and $5.15 \times 10^{2} \mathrm{Cfu} \mathrm{g}^{-1}$, respectively) and it was on par with $50 \% \mathrm{~K}$ through Bio-K $+50 \%$ $\mathrm{K}$ through $\mathrm{K}_{2} \mathrm{SO}_{4}+$ balance NP through fertilizers for targeted yield of $25 \mathrm{t} \mathrm{ha}^{-1}$ (15.15 $\times 10^{6} \mathrm{Cfu} \mathrm{g}^{-1}, 9.23 \times 10^{4} \mathrm{Cfu} \mathrm{g}^{-1}$ and $5.02 \times 10^{2} \mathrm{Cfu} \mathrm{g}^{-1}$, respectively) across all the treatments in potato and finger millet $\left(13.80 \times 10^{5} \mathrm{Cfu} \mathrm{g}^{-1}, 8.36 \times 10^{3} \mathrm{Cfu} \mathrm{g}^{-1}\right.$ and 3.06 x $10^{2} \mathrm{Cfu} \mathrm{g}^{-1}$, respectively) across all the treatments.

\section{Introduction}

Differences in crop yield between different fields in the same region have existed for a long time. Methods for improving the productivity of low-yield fields are a hot topic in large-scale agriculture. Potato plants have shallow root systems and are grown widely in India. In pursuit of higher yields, farmers are applying tremendous amounts of fertilizers, 
which have led to not only lower yield, but low nutrient use efficiency and environmental problems as well. For better improvement of nutrient use efficiency and decreased environmental impacts, improved methods for optimum fertilizer recommendations are necessary for potato crop. Low soil fertility and crop nutrient imbalances are two major obstacles preventing farmers from realizing the higher yields. The extensive nutrient depletion, soil degradation and soil quality deterioration are due to ineffective agricultural practices and inefficient fertilizer application.

Potassium, being associated with the movement of water, nutrients and carbohydrates in plant tissues, activation enzymes affecting protein, starch and adenosine triphosphate (ATP) production, thereby acts as a regulator of rate of photosynthesis, required by the crop in larger quantities and therefore called as a macronutrient.

In many annual crops, potassium has roles in regulation of stomata, which regulates the exchange of water vapour, oxygen and carbon dioxide between plant and environment. Indirectly, this stomata regulation is helpful in maintenance of plant temperature, as many of the reactions in the plant organs are temperature dependant, stunted crop growth and yield reduction are noticeable under deficiency and inadequate supply of this nutrient.

Even though the total $\mathrm{K}$ content of soils exceeds 20,000 ppm (parts per million) in most of the cultivated soils and its supply from the soil is also quite large, very small amounts are available for plant growth at a particular time since most of the soil potassium is locked in the structural component of soil minerals making it unavailable for plant absorption and utilization for growth. Even though it is supplied by external sources like fertilizers, non-scientific recommendations and application methodologies affect its availability to crop. Research conducted by International Plant Nutrition Institute (IPNI) in India clearly shows that guidelines for fertiliser potassium recommendations are inadequate for current yield targets, and as a result the soil test $\mathrm{K}$ level once considered adequate turns out to be insufficient to balance the high rates of $\mathrm{N}$ and $\mathrm{P}$ being applied (Tiwari et al., 2005).

So, there is a need to think on advanced approach to make sure that the potassium is applied as and when the crop requires it and applied potassium is efficiently utilized by the crop. As explained above, site specific nutrient management is the most promising approach for succeeding in this direction.

Among the various methods of fertilizer application approaches, the one based on "yield targeting" (SSNM) are unique in the sense that these methods not only indicates soil test based fertilizer dose but also the level of yield the farmer can hope to achieve if good nutrient management practices are followed in raising the crop. Therefore, considering the importance of the aspects experiments were conducted to study the impact of potato + pigeon pea based intercropping systems on microbial properties.

\section{Materials and Methods}

Field experiments were conducted on the "Impact of potato + pigeon pea based intercropping systems on microbial properties" in the premises of Agricultural Research Station (ARS), Madenur, Hassan Taluk and District during Kharif and Rabi 2017 and 2018.

The experimental plots of kharif season were divided into two plots to raise finger millet and field bean crops with recommended dose 
of fertilizers to study the effect of SSNM on succeeding crops. The treatment details consisting of twelve treatments $\left(\mathrm{T}_{1}: 100 \% \mathrm{~K}\right.$ through $\mathrm{K}_{2} \mathrm{SO}_{4}+\mathrm{NP}$ through fertilizers for targeted yield of $20 \mathrm{t} \mathrm{ha}^{-1,} \mathrm{~T}_{2}: 100 \% \mathrm{~K}$ through Bio-K + balance NP through fertilizers for targeted yield of $20 \mathrm{tha}^{-1,} \mathrm{~T}_{3}: 50$ $\% \mathrm{~K}$ through Bio-K $+50 \% \mathrm{~K}$ through $\mathrm{K}_{2} \mathrm{SO}_{4}$ + balance NP through fertilizers for targeted yield of $20 \mathrm{t} \mathrm{ha}^{-1,} \mathrm{~T}_{4}: 100 \% \mathrm{~K}$ through $\mathrm{K}_{2} \mathrm{SO}_{4}$ + NP through fertilizers for targeted yield of $25 \mathrm{t} \mathrm{ha}^{-1,} \mathrm{~T}_{5}$ : $100 \% \mathrm{~K}$ through Bio-K + balance NP through fertilizers for targeted yield of $25 \mathrm{t} \mathrm{ha}^{-1,} \mathrm{~T}_{6}$ : $50 \% \mathrm{~K}$ through Bio-K + $50 \% \mathrm{~K}$ through $\mathrm{K}_{2} \mathrm{SO}_{4}+$ balance NP through fertilizers for targeted yield of $25 \mathrm{t} \mathrm{ha}^{-1,} \mathrm{~T}_{7}$ : Recommended dose of fertilizers alone (75:75:100 kg NPK ha'), $\mathrm{T}_{8}$ :

Package of Practice recommendation, UAS B) with three replication. The experimental data obtained were subjected to statistical analysis adopting Fisher's method of Analysis of Variance (ANOVA) as outlined by Gomez and Gomez (1984).

The level of significance used for $\mathrm{F}$ test was 5 per cent. Critical difference (CD) values are given in the table at 5 per cent level of significance, wherever the "F" test was significant at 5 per cent level.

Pooled analysis of two years data was analysed using Additive Main Multiplicative Interaction (AMMI) method by Felipe de Mendibur (2020).

\section{Results and Discussion}

The microbial analysis pertaining to bacteria, fungi and actinomycetes population in soil after harvest of potato differed significantly due to supply of nutrients through site specific nutrient management in potato + pigeon pea based inter cropping system and are presented in Table 1. Significantly higher bacterial population was observed in soil after harvest of potato in plot which received $100 \% \mathrm{~K}$ through Bio-K + balance NP through fertilizers for targeted yield of $25 \mathrm{t} \mathrm{ha}^{-1}$ (15.87 x $10^{6} \mathrm{Cfu} \mathrm{g}^{-1}$ ) and it was on par with $50 \% \mathrm{~K}$ through Bio-K $+50 \% \mathrm{~K}$ through $\mathrm{K}_{2} \mathrm{SO}_{4}+$ balance NP through fertilizers for targeted yield of $25 \mathrm{t} \mathrm{ha}^{-1}\left(15.15 \times 10^{6} \mathrm{Cfu} \mathrm{g}^{-1}\right)$ as compared to other treatments.. Significantly lower bacterial population was observed in recommended dose of fertilizers alone $(6.18 \mathrm{x}$ $\left.10^{6} \mathrm{Cfu} \mathrm{g}^{-1}\right)$.

Fungal population was significantly higher with $100 \% \mathrm{~K}$ through Bio-K + balance NP through fertilizers for targeted yield of $25 \mathrm{t} \mathrm{ha}^{-}$ ${ }^{1}\left(9.53 \times 10^{4} \mathrm{Cfu} \mathrm{g}^{-1}\right)$ and it was found to be on par with $50 \% \mathrm{~K}$ through Bio-K + $50 \% \mathrm{~K}$ through $\mathrm{K}_{2} \mathrm{SO}_{4}+$ balance $\mathrm{NP}$ through fertilizers for targeted yield of $25 \mathrm{t} \mathrm{ha}^{-1}(9.23 \mathrm{x}$ $10^{4} \mathrm{Cfu} \mathrm{g}^{-1}$ ) as compared to other treatments.

Significantly lower fungal population was observed in recommended dose of fertilizers alone $\left(4.25 \times 10^{4} \mathrm{Cfu} \mathrm{g}^{-1}\right)$.

Significantly higher actinomycetes population was observed in soil after harvest of potato in plot which received $100 \% \mathrm{~K}$ through Bio-K + balance NP through fertilizers for targeted yield of $25 \mathrm{t} \mathrm{ha}^{-1}\left(5.15 \times 10^{2} \mathrm{Cfu} \mathrm{g}^{-1}\right)$ and it was on par with $50 \% \mathrm{~K}$ through Bio-K +50 $\% \mathrm{~K}$ through $\mathrm{K}_{2} \mathrm{SO}_{4}+$ balance $\mathrm{NP}$ through fertilizers for targeted yield of $25 \mathrm{t} \mathrm{ha}^{-1}(5.02 \mathrm{x}$ $10^{2} \mathrm{Cfu} \mathrm{g}^{-1}$ ) as compared to other treatments.

Significantly lower actinomycetes population was observed in recommended dose of fertilizers alone $\left(2.66 \times 10^{2} \mathrm{Cfu} \mathrm{g}^{-1}\right)$ (Table 1). Similar results were given by Nyawade et al., (2019) in potato crop.

The bacteria, fungi and actinomycetes population in soil after harvest of finger millet due to site specific nutrient management in potato + pigeon pea based inter cropping system are presented in Table 2 . 
Table.1 Microbial population in soil after harvest of potato as influenced by site specific nutrient management in potato + pigeon pea based intercropping cropping system

\begin{tabular}{|c|c|c|c|c|c|c|c|c|c|}
\hline \multirow[t]{2}{*}{ Treatments } & \multicolumn{3}{|c|}{ Bacteria $\left(x 1^{6} \mathrm{Cfu} \mathrm{g}^{-1}\right)$} & \multicolumn{3}{|c|}{ Fungi $\left(x 1^{4} \mathrm{Cfu} \mathrm{g}^{-1}\right)$} & \multicolumn{3}{|c|}{ Actnomycetes $\left(x 1^{2} \mathrm{Cfu} \mathrm{g}^{-1}\right)$} \\
\hline & 2017 & 2018 & Pooled & 2017 & 2018 & Pooled & 2017 & 2018 & Pooled \\
\hline $\mathbf{T}_{1}$ & 7.02 & 7.60 & 7.31 & 4.00 & 4.50 & 4.25 & 3.50 & 4.20 & 3.85 \\
\hline $\mathbf{T}_{2}$ & 8.01 & 9.00 & 8.51 & 6.13 & 6.81 & 6.47 & 3.90 & 4.50 & 4.20 \\
\hline $\mathbf{T}_{3}$ & 9.03 & 10.10 & 9.57 & 6.00 & 6.60 & 6.30 & 4.02 & 4.51 & 4.27 \\
\hline $\mathbf{T}_{4}$ & 11.00 & 11.71 & 11.36 & 7.50 & 8.11 & 7.81 & 4.00 & 4.50 & 4.25 \\
\hline $\mathbf{T}_{5}$ & 15.50 & 16.23 & 15.87 & 9.03 & 10.02 & 9.53 & 4.59 & 5.70 & 5.15 \\
\hline$T_{6}$ & 14.50 & 15.80 & 15.15 & 8.75 & 9.70 & 9.23 & 4.50 & 5.54 & 5.02 \\
\hline $\mathbf{T}_{7}$ & 6.03 & 6.33 & 6.18 & 4.10 & 4.40 & 4.25 & 2.62 & 2.70 & 2.66 \\
\hline $\mathbf{T}_{8}$ & 6.50 & 7.00 & 6.75 & 4.30 & 4.60 & 4.45 & 3.72 & 4.32 & 4.02 \\
\hline F-test & $* *$ & $* *$ & $* *$ & $* *$ & $* *$ & $* *$ & $* *$ & $* *$ & $* *$ \\
\hline S.Em \pm & 0.37 & 0.40 & 0.27 & 0.30 & 0.32 & 0.22 & 0.23 & 0.26 & 0.17 \\
\hline C.D.@ $5 \%$ & 0.11 & 1.20 & 0.78 & 0.90 & 0.45 & 0.63 & 0.70 & 0.80 & 0.51 \\
\hline
\end{tabular}

Legend:

\begin{tabular}{|c|c|}
\hline $\begin{array}{c}\mathrm{T}_{1}: 100 \% \mathrm{~K} \text { through } \mathrm{K}_{2} \mathrm{SO}_{4}+\mathrm{NP} \text { through fertilizers for } \\
\text { targeted yield of } 20 \mathrm{t} \mathrm{ha}^{-1}\end{array}$ & $\begin{array}{c}T_{5}: 100 \% \mathrm{~K} \text { through Bio-K + balance NP through } \\
\text { fertilizers for targeted yield of } 25 \mathrm{tha}^{-1}\end{array}$ \\
\hline $\begin{array}{c}\mathrm{T}_{2}: 100 \% \mathrm{~K} \text { through Bio-K }+ \text { balance NP through fertilizers for } \\
\text { targeted yield of } 20 \mathrm{t} \mathrm{ha}^{-1}\end{array}$ & $\begin{array}{l}\mathbf{T}_{6}: 50 \% \mathrm{~K} \text { through Bio-K }+50 \% \mathrm{~K} \text { through } \mathrm{K}_{2} \mathrm{SO}_{4}+ \\
\text { balance NP through fertilizers for targeted yield of } 25 \mathrm{tha}^{-1}\end{array}$ \\
\hline $\begin{array}{c}\mathrm{T}_{3}: 50 \% \mathrm{~K} \text { through Bio-K }+50 \% \mathrm{~K} \text { through } \mathrm{K}_{2} \mathrm{SO}_{4}+\text { balance } \\
\text { NP through fertilizers for targeted yield of } 20 \mathrm{t} \mathrm{ha}^{-1}\end{array}$ & $\begin{array}{c}\mathbf{T}_{7}: \text { Recommended dose of fertilizers alone }(75: 75: 100 \mathrm{~kg} \\
\left.\text { NPK } \mathrm{ha}^{-1}\right)\end{array}$ \\
\hline $\begin{array}{c}\mathrm{T}_{4}: 100 \% \mathrm{~K} \text { through } \mathrm{K}_{2} \mathrm{SO}_{4}+\mathrm{NP} \text { through fertilizers for } \\
\text { targeted yield of } 25 \mathrm{t} \mathrm{ha}^{-1}\end{array}$ & $\mathbf{T}_{\mathbf{8}}$ : Package of Practice recommendation (UAS B) \\
\hline
\end{tabular}


Table.2 Microbial population in soil after harvest of finger millet as influenced by site specific nutrient management in potato + pigeon pea based intercropping cropping system

\begin{tabular}{|c|c|c|c|c|c|c|c|c|c|}
\hline \multirow[t]{2}{*}{ Treatments } & \multicolumn{3}{|c|}{ Bacteria $\left(x 1^{5} \mathrm{Cfu} \mathrm{g}^{-1}\right)$} & \multicolumn{3}{|c|}{ Fungi $\left(x 1^{3} \mathrm{Cfu} \mathrm{g}^{-1}\right)$} & \multicolumn{3}{|c|}{ Actnomycetes $\left(x 1^{2} \mathrm{Cfu} \mathrm{g}^{-1}\right)$} \\
\hline & 2017 & 2018 & Pooled & 2017 & 2018 & Pooled & 2017 & 2018 & Pooled \\
\hline $\mathbf{T}_{1}$ & 5.27 & 5.70 & 5.48 & 3.50 & 3.60 & 3.55 & 1.30 & 1.40 & 1.35 \\
\hline $\mathbf{T}_{2}$ & 7.70 & 8.52 & 8.11 & 3.02 & 3.30 & 3.16 & 2.80 & 2.73 & 2.77 \\
\hline $\mathbf{T}_{3}$ & 8.22 & 8.64 & 8.43 & 5.00 & 5.43 & 5.22 & 2.43 & 2.52 & 2.48 \\
\hline $\mathbf{T}_{4}$ & 9.20 & 9.70 & 9.45 & 6.51 & 6.80 & 6.66 & 2.04 & 2.30 & 2.17 \\
\hline $\mathbf{T}_{5}$ & 13.20 & 14.40 & 13.80 & 8.01 & 8.70 & 8.36 & 3.00 & 3.12 & 3.06 \\
\hline$T_{6}$ & 12.80 & 13.80 & 13.30 & 7.20 & 8.22 & 7.71 & 2.80 & 3.07 & 2.93 \\
\hline $\mathbf{T}_{7}$ & 4.24 & 4.80 & 4.52 & 2.30 & 2.80 & 2.55 & 1.10 & 1.30 & 1.20 \\
\hline $\mathbf{T}_{8}$ & 4.60 & 4.90 & 4.75 & 2.50 & 3.00 & 2.75 & 1.50 & 1.70 & 1.60 \\
\hline F-test & $* *$ & $* *$ & $* *$ & $* *$ & $* *$ & $* *$ & $* *$ & $* *$ & $* *$ \\
\hline S.Em \pm & 0.41 & 0.50 & 0.32 & 0.31 & 0.25 & 0.20 & 0.18 & 0.16 & 0.12 \\
\hline C.D.@ $5 \%$ & 1.25 & 1.51 & 0.94 & 0.93 & 0.76 & 0.57 & 0.53 & 0.48 & 0.34 \\
\hline
\end{tabular}

Legend:

\begin{tabular}{|c|c|}
\hline $\begin{array}{c}\mathrm{T}_{1}: 100 \% \mathrm{~K} \text { through } \mathrm{K}_{2} \mathrm{SO}_{4}+\mathrm{NP} \text { through fertilizers for } \\
\text { targeted yield of } 20 \mathrm{t} \mathrm{ha}^{-1}\end{array}$ & $\begin{array}{c}T_{5}: 100 \% \mathrm{~K} \text { through Bio-K + balance NP through } \\
\text { fertilizers for targeted yield of } 25 \mathrm{tha}^{-1}\end{array}$ \\
\hline $\begin{array}{c}\mathrm{T}_{2}: 100 \% \mathrm{~K} \text { through Bio-K }+ \text { balance NP through fertilizers for } \\
\text { targeted yield of } 20 \mathrm{t} \mathrm{ha}^{-1}\end{array}$ & $\begin{array}{l}\mathbf{T}_{6}: 50 \% \mathrm{~K} \text { through Bio-K }+50 \% \mathrm{~K} \text { through } \mathrm{K}_{2} \mathrm{SO}_{4}+ \\
\text { balance NP through fertilizers for targeted yield of } 25 \mathrm{tha}^{-1}\end{array}$ \\
\hline $\begin{array}{c}\mathrm{T}_{3}: 50 \% \mathrm{~K} \text { through Bio-K }+50 \% \mathrm{~K} \text { through } \mathrm{K}_{2} \mathrm{SO}_{4}+\text { balance } \\
\text { NP through fertilizers for targeted yield of } 20 \mathrm{t} \mathrm{ha}^{-1}\end{array}$ & $\begin{array}{c}\mathbf{T}_{7}: \text { Recommended dose of fertilizers alone }(75: 75: 100 \mathrm{~kg} \\
\left.\text { NPK } \mathrm{ha}^{-1}\right)\end{array}$ \\
\hline $\begin{array}{c}\mathrm{T}_{4}: 100 \% \mathrm{~K} \text { through } \mathrm{K}_{2} \mathrm{SO}_{4}+\mathrm{NP} \text { through fertilizers for } \\
\text { targeted yield of } 25 \mathrm{t} \mathrm{ha}^{-1}\end{array}$ & $\mathbf{T}_{\mathbf{8}}$ : Package of Practice recommendation (UAS B) \\
\hline
\end{tabular}


Table.3 Microbial population in soil after harvest of field bean as influenced by site specific nutrient management in potato + pigeon pea based intercropping cropping system

\begin{tabular}{|c|c|c|c|c|c|c|c|c|c|}
\hline \multirow[t]{2}{*}{ Treatments } & \multicolumn{3}{|c|}{ Bacteria $\left(x 1^{6} \mathrm{Cfu}^{-1}\right)$} & \multicolumn{3}{|c|}{ Fungi $\left(\times 10^{4} \mathrm{Cfu} \mathrm{g}^{-1}\right)$} & \multicolumn{3}{|c|}{ Actnomycetes $\left(\times 1^{2} \mathrm{Cfu} \mathrm{g}^{-1}\right)$} \\
\hline & 2017 & 2018 & Pooled & 2017 & 2018 & Pooled & 2017 & 2018 & Pooled \\
\hline $\mathbf{T}_{1}$ & 9.54 & 11.01 & 10.28 & 6.03 & 7.10 & 6.57 & 4.20 & 5.00 & 4.60 \\
\hline $\mathbf{T}_{2}$ & 12.00 & 13.80 & 12.90 & 6.50 & 7.83 & 7.17 & 4.01 & 5.43 & 4.72 \\
\hline $\mathbf{T}_{3}$ & 13.53 & 13.90 & 13.72 & 7.50 & 8.90 & 8.20 & 4.42 & 5.80 & 5.11 \\
\hline $\mathbf{T}_{4}$ & 13.50 & 14.04 & 13.77 & 8.00 & 9.30 & 8.65 & 4.53 & 6.31 & 5.42 \\
\hline $\mathbf{T}_{5}$ & 17.50 & 18.70 & 18.10 & 10.50 & 11.21 & 10.86 & 6.00 & 6.80 & 6.40 \\
\hline$T_{6}$ & 16.92 & 18.21 & 17.57 & 10.00 & 10.72 & 10.36 & 5.81 & 6.12 & 5.97 \\
\hline $\mathbf{T}_{7}$ & 8.50 & 10.11 & 9.31 & 5.80 & 6.30 & 6.05 & 3.10 & 3.60 & 3.35 \\
\hline $\mathbf{T}_{8}$ & 9.80 & 11.50 & 10.65 & 7.00 & 8.50 & 7.75 & 4.50 & 5.43 & 4.97 \\
\hline F-test & $* *$ & $* *$ & $* *$ & $* *$ & $* *$ & $* *$ & $* *$ & $* *$ & $* *$ \\
\hline S.Em \pm & 0.53 & 0.54 & 0.38 & 0.34 & 0.39 & 0.26 & 0.27 & 0.31 & 0.21 \\
\hline C.D.@ 5\% & 1.62 & 1.63 & 1.10 & 1.02 & 1.19 & 0.75 & 0.81 & 0.95 & 0.60 \\
\hline
\end{tabular}

Legend:

\begin{tabular}{|c|c|}
\hline $\begin{array}{c}\mathrm{T}_{1}: 100 \% \mathrm{~K} \text { through } \mathrm{K}_{2} \mathrm{SO}_{4}+\mathrm{NP} \text { through fertilizers for } \\
\text { targeted yield of } 20 \mathrm{t} \mathrm{ha}^{-1}\end{array}$ & $\begin{array}{c}T_{5}: 100 \% \mathrm{~K} \text { through Bio-K + balance NP through } \\
\text { fertilizers for targeted yield of } 25 \mathrm{tha}^{-1}\end{array}$ \\
\hline $\begin{array}{c}\mathrm{T}_{2}: 100 \% \mathrm{~K} \text { through Bio-K }+ \text { balance NP through fertilizers for } \\
\text { targeted yield of } 20 \mathrm{t} \mathrm{ha}^{-1}\end{array}$ & $\begin{array}{l}\mathbf{T}_{6}: 50 \% \mathrm{~K} \text { through Bio-K }+50 \% \mathrm{~K} \text { through } \mathrm{K}_{2} \mathrm{SO}_{4}+ \\
\text { balance NP through fertilizers for targeted yield of } 25 \mathrm{tha}^{-1}\end{array}$ \\
\hline $\begin{array}{c}\mathrm{T}_{3}: 50 \% \mathrm{~K} \text { through Bio-K }+50 \% \mathrm{~K} \text { through } \mathrm{K}_{2} \mathrm{SO}_{4}+\text { balance } \\
\text { NP through fertilizers for targeted yield of } 20 \mathrm{t} \mathrm{ha}^{-1}\end{array}$ & $\begin{array}{c}\mathbf{T}_{7}: \text { Recommended dose of fertilizers alone }(75: 75: 100 \mathrm{~kg} \\
\left.\text { NPK } \mathrm{ha}^{-1}\right)\end{array}$ \\
\hline $\begin{array}{c}\mathrm{T}_{4}: 100 \% \mathrm{~K} \text { through } \mathrm{K}_{2} \mathrm{SO}_{4}+\mathrm{NP} \text { through fertilizers for } \\
\text { targeted yield of } 25 \mathrm{t} \mathrm{ha}^{-1}\end{array}$ & $\mathbf{T}_{\mathbf{8}}$ : Package of Practice recommendation (UAS B) \\
\hline
\end{tabular}


Significantly higher bacterial population was observed in soil after harvest of finger millet in plot which received $100 \% \mathrm{~K}$ through Bio$\mathrm{K}+$ balance NP through fertilizers for targeted yield of $25 \mathrm{t} \mathrm{ha}^{-1}\left(13.80 \times 10^{5} \mathrm{Cfu} \mathrm{g}^{-1}\right)$ and it was on par with $50 \% \mathrm{~K}$ through Bio-K +50 $\% \mathrm{~K}$ through $\mathrm{K}_{2} \mathrm{SO}_{4}+$ balance $\mathrm{NP}$ through fertilizers for targeted yield of $25 \mathrm{t} \mathrm{ha}^{-11}$ (13.30 $\mathrm{x} 10^{5} \mathrm{Cfu} \mathrm{g}^{-1}$ ) as compared to other treatments. Significantly lower bacterial population was observed in recommended dose of fertilizers alone $\left(4.52 \times 10^{5} \mathrm{Cfu} \mathrm{g}^{-1}\right)$.

Fungal population was significantly higher with $100 \% \mathrm{~K}$ through Bio-K + balance NP through fertilizers for targeted yield of $25 \mathrm{tha}^{-}$ ${ }^{1}\left(8.36 \times 10^{3} \mathrm{Cfu} \mathrm{g}^{-1}\right)$ as compared to other treatments. Significantly lower fungal population was observed in recommended dose of fertilizers alone $\left(2.55 \times 10^{3} \mathrm{Cfu} \mathrm{g}^{-1}\right)$.

Significantly higher actinomycetes population was observed in soil after harvest of potato in plot which received $100 \% \mathrm{~K}$ through Bio-K + balance NP through fertilizers for targeted yield of $25 \mathrm{t} \mathrm{ha}^{-1}\left(3.06 \times 10^{2} \mathrm{Cfu} \mathrm{g}^{-1}\right)$ and it was on par with $50 \% \mathrm{~K}$ through Bio-K +50 $\% \mathrm{~K}$ through $\mathrm{K}_{2} \mathrm{SO}_{4}+$ balance $\mathrm{NP}$ through fertilizers for targeted yield of $25 \mathrm{tha}^{-1}(2.93 \mathrm{x}$ $10^{2} \mathrm{Cfu} \mathrm{g}^{-1}$ ) and $100 \% \mathrm{~K}$ through Bio-K + balance NP through fertilizers for targeted yield of $20 \mathrm{t} \mathrm{ha}^{-1}\left(2.77 \times 10^{2} \mathrm{Cfu} \mathrm{g}^{-1}\right)$ as compared to other treatments. Significantly lower actinomycetes population was observed in recommended dose of fertilizers alone (1.20 $\mathrm{x} 10^{2} \mathrm{Cfu} \mathrm{g}^{-1}$ ) (Table 2). The results are in accordance with studies of Umesh (2008) in pigeon pea and Lingaraju (2018) in cowpea.

Significantly higher bacterial population was observed in soil after harvest of field bean in plot which received $100 \% \mathrm{~K}$ through Bio-K + balance NP through fertilizers for targeted yield of $25 \mathrm{t} \mathrm{ha}^{-1}\left(18.10 \times 10^{6} \mathrm{Cfu} \mathrm{g}^{-1}\right)$ and it was on par with $50 \% \mathrm{~K}$ through Bio-K +50 $\% \mathrm{~K}$ through $\mathrm{K}_{2} \mathrm{SO}_{4}+$ balance $\mathrm{NP}$ through fertilizers for targeted yield of $25 \mathrm{t} \mathrm{ha}^{-1}$ (17.57 $\mathrm{x} 10^{6} \mathrm{Cfu} \mathrm{g}^{-1}$ ) as compared to other treatments.. Significantly lower bacterial population was observed in recommended dose of fertilizers alone $\left(9.31 \times 10^{6} \mathrm{Cfu} \mathrm{g}^{-1}\right)$ (Table 3).

Fungal population was significantly higher with $100 \% \mathrm{~K}$ through Bio-K + balance NP through fertilizers for targeted yield of $25 \mathrm{t} \mathrm{ha}^{-}$ ${ }^{1}\left(10.86 \times 10^{4} \mathrm{Cfu} \mathrm{g}^{-1}\right)$ and it was found to be on par with $50 \% \mathrm{~K}$ through Bio-K $+50 \% \mathrm{~K}$ through $\mathrm{K}_{2} \mathrm{SO}_{4}+$ balance $\mathrm{NP}$ through fertilizers for targeted yield of $25 \mathrm{t} \mathrm{ha}^{-1}$ (10.36 x $\left.10^{4} \mathrm{Cfu} \mathrm{g}^{-1}\right){ }^{\text {as }}$ compared to other treatments.

Significantly lower fungal population was observed in recommended dose of fertilizers alone $\left(6.05 \times 10^{4} \mathrm{Cfu} \mathrm{g}^{-1}\right)$ (Table 3).

Significantly higher actinomycetes population was observed in soil after harvest of field bean in plot which received $100 \% \mathrm{~K}$ through Bio$\mathrm{K}+$ balance NP through fertilizers for targeted yield of $25 \mathrm{t} \mathrm{ha}^{-1}\left(6.40 \times 10^{2} \mathrm{Cfu} \mathrm{g}^{-1}\right)$ and it was on par with $50 \% \mathrm{~K}$ through Bio-K +50 $\% \mathrm{~K}$ through $\mathrm{K}_{2} \mathrm{SO}_{4}+$ balance $\mathrm{NP}$ through fertilizers for targeted yield of $25 \mathrm{t} \mathrm{ha}^{-1}$ (5.97 x $10^{2} \mathrm{Cfu}^{-1}$ ) as compared to other treatments. Significantly lower actinomycetes population was observed in recommended dose of fertilizers alone $\left(3.35 \times 10^{2} \mathrm{Cfu} \mathrm{g}^{-1}\right)$.

This increased microorganisms population might be attributed to increased availability of macro and micronutrients during the crop growth period due to addition of organic manure along with macronutrients and micro nutrients. Nitrogen fixation through free leaving nitrogen fixing rhizobium and mobilization of $\mathrm{P}$ and micronutrients along with addition of leaf litter and crop residues into the soil during reproductive stage which might have favoured increased population of microorganisms. Similar results were reported by Gitari et al., (2019). 
In potato + pigeon pea inter cropping system, after the potato harvesting, field bean showed higher microbial population under SSNM approach. This provides greater crop selections and sowing windows to the potato growing farmers of the zone.

\section{References}

Felipe De Mendibur, 2020, Agricolae: Statistical procedures for agricultural research. R package version 1.3-3.

Gitari, Harun I, Charles, K.K., Gachene, Nancy, N., Karnja, Solomon KAMAU, Shadrack Nyawade and Elmar Schulte Geldermann,2019, Potato-legume intercropping on a sloping terrain and its effects on soil physic-chemical properties. Plant Soil,440 (1-2):327335.

Gomez, K. A. and Gomez, A. A., 1984, Statistical procedures for Agricultural Research, 2 ${ }^{\text {nd }}$ Edition A Wiley Inter Science Publication. New York (USA). Lingaraju, N. N., 2018, response of maizecowpea sequence to treated sugar mill effluent irrigation under different nutrient and moisture regimes in Cauvery command area. Ph.D. Thesis, University of Agricultural Sciences, Bangalore.

Nyawade, Shadrack, O, Nancy, N. Karanja, Charles, K. K., Gachene, Harun, Elmar Schulte-Geldermann and Monica Parker, 2019, Short term dynamics of soil organic matter fractions and microbial activity in smallholder potato-legume intercropping systems. Applied Soil Ecology, 142: 123-135.

Tiwari, K. N., Dwivedi, B. S., Singh, D., Swarup, A., Meena, M. C., Majumdar, K., Yadav, K. S. and Yadav, R. L., 2005, On-farm evaluation of SSNM in pearl millet based cropping systems on alluvial soils. Better Crops, 3(1): 2527.

Umesh, M. R., 2008, Investigation on balanced fertilization for maize pigeon pea cropping sequence in Alfisols of Karnataka. Ph.D. Thesis. University of Agricultural Sciences, Bengaluru.

\section{How to cite this article:}

Manjunatha, M. H., S. Bhaskar, A. Sathish, B. Basavaraja and Lingaraju, N. N. 2021. Impact of Potato + Pigeon Pea based Intercropping Systems on Microbial Properties. Int.J.Curr.Microbiol.App.Sci. 10(02): 1745-1752.

doi: https://doi.org/10.20546/ijcmas.2021.1002.206 